\title{
SET-SET TOPOLOGIES AND SEMITOPOLOGICAL GROUPS
}

\section{KATHRYN F. PORTER}

Department of Mathematical Sciences

Ba 11 St ate University

Muncie, IN 47306-0490

(Received May 10, 1988)

ABSTRACT. Let $G$ be a group with binary operation, $:$ Let $T$ be a topology for $G$ such that for all $g \in G$ the maps, $m_{g}: G+G$ and $g: G+G$, defined by $m_{g}(f)=f \cdot g$ and $g^{m(f)}=g \cdot f$, respectively, are continuous. Then $(G, T)$ is called a semitopological group. Some specific set-set topologies for function spaces are discussed and the concept of topologically determined collections of sets is introduced and used to classify some set-set topologies as semitopological groups.

KEY WORDS AND PHRASES. Point-open topology, compact-open topology, g-topology, Btopology, topologically determined collection of sets. 1980 AMS SUBJECT CLASSIFICATION CODES. Primary 54C35, 57SO5. Secondary $54 \mathrm{H} 9$.

1. INTRODUCTION.

Husain [1] defined a semitopological group as a group, $G$, with a binary operation,., and a topology, T, such that both right and left "multiplication" are continuous. We shall define the concept of topologically determined collections of sets and present some results which help classify some set-set topologies on function spaces as semitopological groups.

2. SOME TOPOLOGIES FOR FUNCTION SPACES.

Before beginning our discussion of semitopological groups, we present the definitions of some topologies for function spaces to which we will be referring.

DEFINITION 1. [2] Let $(X, T)$ and $(Y, \tilde{T})$ be topological spaces. For $p \in X$ and for $U \in \tilde{T}$, define the set $(\{p\}, U)=\left\{f \in Y^{X}: f(p) \in U\right\}$. The $n$, we define $S=\{(\{p\}, U): P \in X$ and $U \in \tilde{T}\}$. $S$ is a subbasis for a topology, $T_{p}$, on $Y^{X}$, called the point-open topology.

DEFINITION 2. Let $(X, T)$ and $(Y, \tilde{T})$ be topological spaces. For $K \subseteq X$ and for $U \subseteq$ $Y$, define the set $(K, U)=\left\{f \in Y^{X}: f(K) \subseteq U\right\}$. Next, we define the sets $S_{c o}=\{(K, U): K$ is a compact subset of $X$ and $U \in \tilde{T}\}$ and $S_{g}=\{(K, U)$ : $\mathrm{U} \in \tilde{\mathrm{T}}$ and $(\mathrm{X} \backslash \mathrm{K}) \in \mathrm{T}$, and either $\mathrm{K}$ or $(\mathrm{Y} \backslash \mathrm{U})$ is compact\}. Then $\mathrm{S}_{\mathrm{co}}$ and $\mathrm{S}_{\mathrm{g}}$ are subbases for topologies, $\mathrm{T}_{\text {co }}$ and $\mathrm{T}_{\mathrm{g}}$, respectively, on $\mathrm{Y}^{\mathrm{X}}$. $\mathrm{T}_{\mathrm{co}}$ is called the compact-open topology [2], while $\mathrm{T}_{\mathrm{g}}$ is named the g-topology [3].

These three topologies are related as follows: $T_{p} \subseteq T_{\text {co }}$ and if $\mathrm{X}$ is $\mathrm{T}_{2}$, then $\mathrm{T}_{\mathrm{co}} \subseteq \mathrm{T}_{\mathrm{g}} \cdot \quad \mathrm{T}_{2}$ is needed so that compact sets in $\mathrm{X}$ are closed. 
DEFINITION 3. Let $(X, T)$ and $(Y, \widetilde{T})$ be topological spaces. For $U \subseteq X$ and $V \subseteq Y$, define the $\operatorname{set} B(U, V)=\left\{f \in Y^{X}: f(U) \cap V \neq \emptyset\right\}$. Then we set

$S=\{B(U, V): U \in T$ and $V \in \tilde{T}\}$. Then $S$ is a subbasis for a topology on $Y^{X}$ called the $B$ topology which will be denoted by $T_{B}$.

THEOREM 1. Let $(X, T)$ and $(Y, \tilde{T})$ be topological spaces and let $F \subseteq C(X, Y)$. Then $T_{B} \subseteq T_{p}$.

PROOF. Let $B(U, V)$ be a subbasic open set in $T_{B}$.

Then $B(U, V)=\underset{x \in U}{U}(\{x\}, V)$ and $\underset{x \in U}{U}(\{x\}, V) \in T_{p} \cdot \quad$ Thus, $T_{B} \subseteq T_{p}$.

Note that if $X$ is discrete or $T_{p}$ is trivial, then $T_{p}=T_{B}$. Howeve $r$, the converse of this statement is not true as Example 1 will show.

EXAMP LE 1. Let $\mathrm{X}=\{1,2,3,4\}$. Let $\mathrm{T}=\{0, \mathrm{X},\{1,2\},\{3,4\}\}$ be a topology on $\mathrm{X}$. $\mathrm{T}$ is obviously not discrete. ( $\{1\},\{3,4\})$ and $(\{3\},\{3,4\})$ are non-empty subbasic proper open sets in $\left(C(X, X), T_{p}\right)$ and $(\{1\},\{3,4\}) \neq(\{3\},\{3,4\})$.

So we see that $T_{p}$ is not trivial. But $T_{B}=T_{p}$.

EXAMPLE 2. Let $X=Z_{+}$with the cofinite topology, $T_{\text {cof }}$, i.e., $0 \in T_{\text {cof }}$ if and only if either $X \backslash 0$ is finite or $X=\phi$. So, if $0, V \in T_{\text {cof }}$ are not empty, $O$ and $V$ are infinite and there exists $j \in X$ such that for all $k>j, k \in 0 \cap v . \quad B(0, V)=H(X)$ if $O$ and $V$ are non-empty. $\left(H(X), T_{B}\right)$ is trivial. But $\left(H(X), T_{p}\right)$ is not, since not every homeomorphism belongs to $(\{1\},\{10,11,12, \ldots\})$. So we see that $T_{B}$ is not always the same as $\mathrm{T}_{\mathrm{p}}$.

3. SEMI TOPOLOGICAL GROUPS.

Note, before we continue, that $H(X)$ is a group with binary operation, 0 , composition, and identity map, $e(x)=x$, for every $x \in X$.

DEFINITION 4. Let $G$ be a group with binary operation, o. A topology $T$, for $G$ is called $\underline{R M C}$ (LMC) provided that, for every $g \in G$, the map $m_{g}: G \rightarrow G, \quad(g m: G \rightarrow G)$, defined by $m_{g}(f)=$ fog, $\left(g^{m}(f)=g \circ f\right)$, is continuous. Here, "RMC" stands for "right multiplication continuous" and "LMC" for "left multiplication continuous."

The topology of uniform convergence, $T \tilde{U}$, on a subgroup of $H(X)$, is always $R M C$ and is LMC under certain conditions. This topology will give us an example of a topology which is not both RMC and LMC.

THEOREM 2. Let $(X, U)$ be a uniform space. Let $G$ be a subgroup of $H(X)$. Let $\tilde{U}$ be the induced uniformity on $\mathrm{G}$. Then $\mathrm{T}_{\tilde{U}}$ is $\mathrm{RMC}$.

PROOF. Recall, given a uniform space, $(X, U)$ for each $U \in U$, define the set, $\tilde{U}=\{(f, g):(f(x), g(x)) \in U$ for all $1 x \in X\}$. Set $B=\{\tilde{U}: U \in U\}$. Then $B$ is a basis for a uniformity on $G, \tilde{U}$, which in turn induces a topology, $T_{\tilde{U}}$, on $G$.

Assume $g \in G$ and 1 et 0 be open in $T_{\tilde{U}^{-}}$Let $f \in \mathrm{m}_{\mathrm{g}}{ }^{-1}(0)$, then fog $\in 0$. Hence there exists $U \in U$ such that $f \circ g \in \tilde{U}[f \circ g] \subseteq 0 . f \in \tilde{U}[f]$. Now if

$h \in \tilde{U}[f],(f(x), h(x)) \in U$, for all $x \in X$. Thus, $(f \circ g(x), h \circ g(x)) \in U$, for $a 11 \quad x \in X$. Hence, $h$ og $g \tilde{U}[f \circ g] \subseteq 0$, and we have that $h \in m_{g}^{-1}(0) . \quad \tilde{U}[f] \subseteq m_{g}^{-1}(0)$.

Thus, $\mathrm{T}_{\tilde{U}}$ is RMC.

DEFINITION 5. [1] Let $G$ be a subgroup of $H(X)$. Let $T$ be a topology for $G$ such that $T$ is both LMC and RMC. Then $(G, T)$ is called a semitopological group. We will denote this by STG. 
THEOREM 3. Let $(X, U)$ be a uniform space. Let $G$ be a subgroup of $H(X)$. Let $\check{U}$ be the induced uniformity on $G$. The $n$, if $g \in G$ implies that $g$ is uniformly continuous w.r.t. $U$, then $\left(G, T_{U}\right)$ is a STG.

PROOF. As sume $g \in G$ and 0 is open in $G$. Let $f \in g^{m}(0)$. Then $g \circ f \in 0$. Hence there exists a $U \in U$ such that $g \circ f \in \tilde{U}[g \circ f]=\{h \in G:(g \cap f, h) \in \tilde{U}\} \subseteq 0$. By definition of uniform continuity, there exists $V \in U$ such that if $(p, q) \in V$ then $(g(p), g(q)) \in U$. Set $W \quad=\quad U \cap V \in U . \quad$ Then $g \circ f \in \tilde{W}[g \circ f] \subseteq \tilde{U}[g \circ f] \subseteq 0 . \quad f \in \tilde{W}[f] . \quad$ Claim: $\tilde{W}[f] \subseteq g^{m}(0)$. If $h \in \tilde{W}[f]$, then $(f(x), h(x)) W \subseteq V$ for all $x \in X$. So, $(g \circ f(x), g \circ h(x)) \in U$ for all $x \in X$. Hence goh $\in \tilde{U}[g \circ f] \in 0$, which means our claim is true and hence $T_{\tilde{U}}$ is LMC. By Theorem 2, $\mathrm{T}_{\tilde{U}}$ is $R M C$, so $(G, T \tilde{U})$ is a STG.

EXAMPLE 3. Let $X=\mathbb{R}$, the reals with the usual unfform structure, 1.e., for each $\varepsilon>0$, we have the basis element $U_{\epsilon}=\{(x, y):|x-y|<\varepsilon\}$. Then a basis for the induced unfformity on $H(X)$ is the collection of all sets of the form, $\tilde{U}_{\epsilon}=\{(f, g):(f(x), g(x)) \in U \in$ for $a 11 x \in X\}$.

Let $g(x)=x^{3}$ and let $e(x)=x$. Let $\varepsilon>0$ be given. The $n$

since goe $=g, e \in_{g} m^{-1}\left(\tilde{U}_{\epsilon}[g]\right)$. Now let $\delta$ be a positive number, so $e \in \tilde{U}_{\delta}[e]$. The $n$ define $h(x)=x+\frac{1}{2} \delta$. Hence, $h \in \tilde{U}_{\delta}[e]$. But goh $(x)=\left(x+\frac{1}{2} \delta\right)^{3}$, which gives that $|g \circ h(x)-g(x)|=\left|\frac{3}{2} \delta_{x}^{2}+\frac{3}{4} \delta^{2} x+\frac{1}{8} \delta^{3}\right|$ and this function has no maximum on $\mathbb{R}$, hence $h \notin g \mathrm{~m}^{-1}\left(\tilde{U}_{\epsilon}[g]\right)$. So, $g^{m}$ is not continuous. $T_{\tilde{U}}$ is not LMC.

4. TOPOLOGICALLY DETERMINEd COLLECTIONS OF SETS.

DEFINITION 6. Let $X$ be a topological space. Let $0 \subseteq P(X)$, the collection of all subsets of $X$, with the property that for each $f \in H(X)$, if $A \in 0$ then $f(A) \in 0$. Then 0 is a topologically determined (TD) collection of sets.

THEOREM 4. Let $(X, T)$ be a topological space and let $\tilde{U}$ and $\tilde{V}$ be collections of subsets of $X$. Let $G$ be subgroup of $H(X)$. Let $S(\tilde{U}, \tilde{V})=\{(U, V): U \in \tilde{U}$ and $V \in \tilde{V}\}$ where $(U, V)=\{f \in G: f(U) \subseteq V\}$. If $S(\tilde{U}, \tilde{V})$ is a subbasis for a topology, $T(\tilde{U}, \tilde{V})$, on $G$, and if $\tilde{U}$ and $\tilde{V}$ are $T D$ collections of sets then $(G, T(\tilde{U}, \tilde{V})$ ) is a semitopological group.

PROOF. Let $(U, V)$ be a subbasic open set in $T(\tilde{U}, \tilde{V})$ and let $f \in G$. As sume $g \in m_{f}^{-1}(U, V)$ and $h \in f^{m^{-1}}((U, V))$. Then $g \circ f(U) \subseteq V$ and $f \circ h(U) \subseteq V$. $g \circ f(U) \subseteq V$ implies $g \in(f(U), V) \in T(\tilde{U}, \tilde{V})$ since $\tilde{U}$ is $T D$. Suppose that $\theta \in(f(U), V)$ then $\theta$ of $(U) \subseteq V$. So $\theta \in m_{f}^{-1}((U, V))$. Therefore, $(f(U),(V)) \subseteq$ $m_{f}^{-1}((U, V))$, which shows that $m_{f}$ is continuous.

fo $h(U) \subseteq V$ gives us that $h \in\left(U, f^{-1}(V)\right) \in T(\tilde{U}, \tilde{V})$ since $\tilde{V}$ is $T D$. Notice that if $\gamma \in\left(U, f^{-1}(V)\right)$ then $\gamma(U) \subseteq f^{-1}(V)$, so $\gamma \in_{f} m^{-1}((U, V))$.

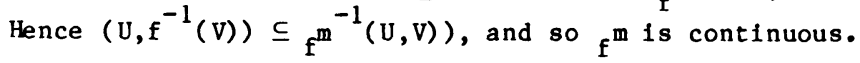

Note from the proof of Theorem 4, that if $(G, T(\tilde{U}, \tilde{V}))$ is as defined in Theorem 4, then if $\tilde{U}$ is $T D$, we have that $(\tilde{U}, \tilde{V})$ is RMC. Simllarly, if $\tilde{V}$ is $T D$ then $T(\tilde{U}, \tilde{V})$ is $L M C$.

Some of the TD collections of sets for a topological space, $X$, are:

1) all the open subsets of $X$

ii) all the closed subsets of $x$

iii) a 11 the compact subsets of $X$ 
iv) all the singleton subsets of $X$

v) all the connected subsets of $X$

vi) all the regular open subsets of $X$

vii) all the regular closed subsets of $X$

Considering the above list, we have the following:

COROLLARY 4.1. Let $X$ be a topological space and let $G$ be a subgroup of $H(X)$. Then $(G, T)$ is a STG when $T=T_{p}$ or $T_{c o}$.

PROOF. Theorem 4 along with 1 , 111 , and iv above give us the desired conclusion.

THEOREM 5. If $U_{1}, U_{2}, V_{1}$, and $V_{2}$ are TD collections of subsets of $X$, then $(G, T)$ is a STG where $T$ is the smallest topology containing both $T\left(U_{1}, V_{1}\right)$ and

$T\left(U_{2}, V_{2}\right)$. (We denote this by $T=T\left(U_{1}, V_{1}\right) \vee T\left(U_{2}, V_{2}\right) \cdot$ )

PROOF. T has as a subbasis, $S$, the union of the subbases $S\left(U_{1}, V_{1}\right)$ and $S\left(U_{2}, V_{2}\right)$ of $\mathrm{T}\left(\mathrm{U}_{1}, \mathrm{~V}_{1}\right)$ and $\mathrm{T}\left(\mathrm{U}_{2}, \mathrm{~V}_{2}\right)$ respectively. So if $(\mathrm{U}, \mathrm{V}) \in \mathrm{S}$ then either $(\mathrm{U}, \mathrm{V}) \in \mathrm{S}\left(\mathrm{U}_{1}, \mathrm{~V}_{1}\right)$ or $(U, V) \in S\left(U_{2}, V_{2}\right)$, from which our conclusion follows.

COROLlaRY 5.1 Let $(\mathrm{X}, \mathrm{T})$ be a $\mathrm{T}_{2}$ topological space. Let $\mathrm{G}$ be a subgroup of $H(X)$. Then $\left(G, T_{g}\right)$ is a STG.

PROOF. Note $\mathrm{T}_{C O}=\mathrm{T}(\tilde{\mathrm{K}}, \tilde{0})$ where $\tilde{\mathrm{K}}=\{\mathrm{K} \in \mathrm{P}(\mathrm{X}): \mathrm{K}$ is compact $\}$ and $\tilde{0}=\mathrm{T}$. Define $T_{h}=T(\tilde{C}, \tilde{U})$ where $\tilde{C}=\{C \in P(X): C$ is closed $\}$ and $\tilde{U}=\{U \in T:$ (X\U is compact\}. Then $T_{g}=T_{c o} \vee T_{h}$. Since $\tilde{K}, \tilde{0}, \tilde{C}$, and $\tilde{U}$ are $T D$, we immediately obtain from Theorem 5 that $\mathrm{T}_{\mathrm{g}}$ is a STG.

THEOREM 6. Let $(X, T)$ be a topological space and let $G$ be a subgroup of $H(X)$. Let $\tilde{U}, \tilde{V}$ be collections of subsets of $X$. Define, for $U \in \tilde{U}$ and $V \in \tilde{V}$, the set $B(U, V)=\{f \in G: f(U) \cap V \neq \phi\}$ and let $S(B(\tilde{U}, \tilde{V}))=\{B(U, V): U \in \tilde{U}$ and $V \in \tilde{V}\}$. If, $S(B(\tilde{U}, \tilde{V}))$ is a subbasis for a topology, $T(B(\tilde{U}, \tilde{V}))$, on $G$, and if $\tilde{U}$ and $\tilde{V}$ are TD then $(G, T(B(\tilde{U}, \tilde{V}))$ is a STG.

PROOF. Let $B(U, V)$ be a subbasic open set in $G$ and let $f \in G$. As sume that $g \in m_{f}^{-1}(B(U, V))$. By definition of $B(U, V)$, we know that this means that $f \circ g(U) \cap V \neq \phi_{0} \quad$ So, $g(U) \cap f^{-1}(V)=\phi$. He nce, $\left.g \in B\left(U, f^{-1}(V)\right) \subseteq m_{f} B(U, V)\right)$. Now assume $g \in \mathrm{f}^{\mathrm{m}^{-1}}(B(U, V))$, then $g \circ f(U) \cap V \neq 0$. Thus, $g \in B(f(U), V) \subseteq m_{f}^{-1}(B(U, V))$.

From this we have:

COROLLARY 6.1 Let $(X, T)$ be a topological space and let $G$ be a subgroup of $H(X)$. Then $\left(G, T_{B}\right)$ is a STG.

\section{REFERENCES}

1. HUSAIN, T., Introduction to Topological Grulips, 19.66, W.B. Saunders Co., Philadelphia and London.

2. MUNKRES, J.R., Topology A F1rst Course, 1975, Prentice-Ha 11, Englewood C11ffs, New Jersey.

3. ARENS, R.F., "Topologies for Homeomorphism Groups," Ame r. J. Ma th., 68 (1946), pp. 593-610.

4. FOX, R.H., "Topologies for Function Spaces," Bull. Am. Math. Soc. 51 (1945), 429-432.

5. PORTER, K.F., "Evaluation Maps On Groups of Self-Homeomorphisms", Ph.D. Dissertation, 1987, University of Delaware. 


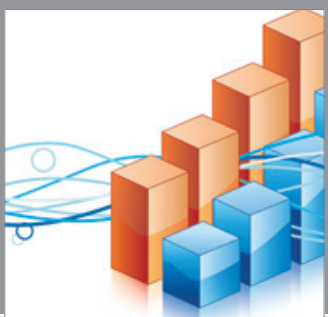

Advances in

Operations Research

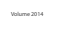

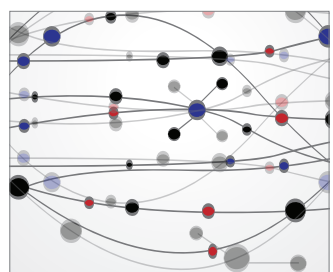

\section{The Scientific} World Journal
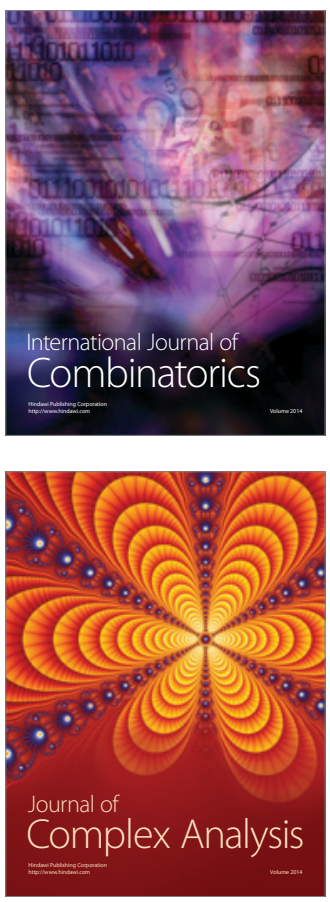

International Journal of

Mathematics and

Mathematical

Sciences
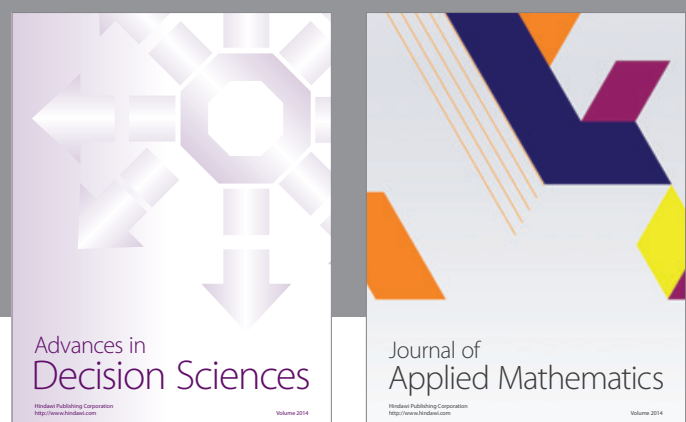

Journal of

Applied Mathematics
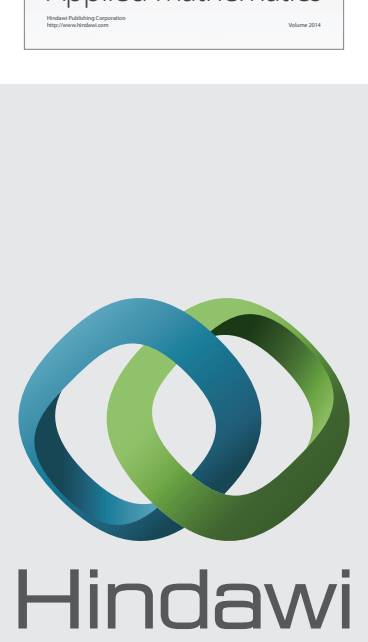

Submit your manuscripts at http://www.hindawi.com
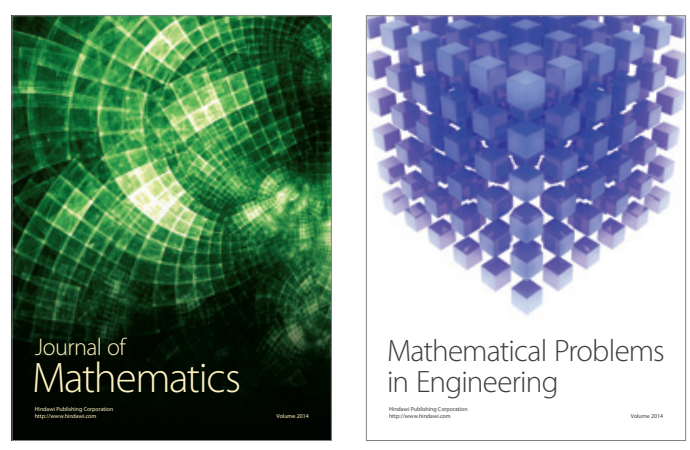

Mathematical Problems in Engineering
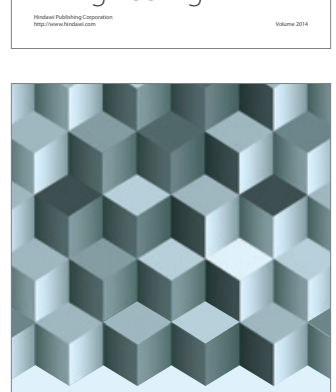

Journal of

Function Spaces
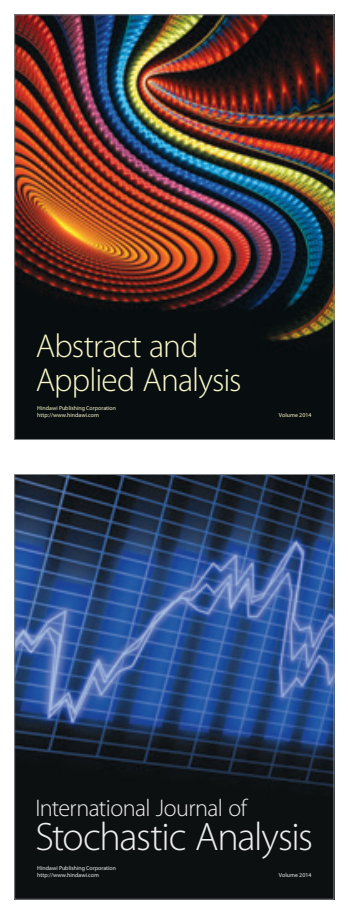

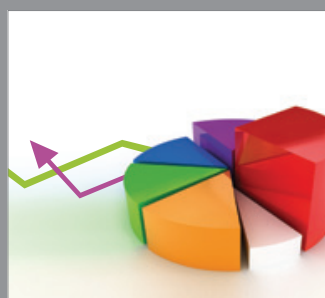

ournal of

Probability and Statistics

Promensencen
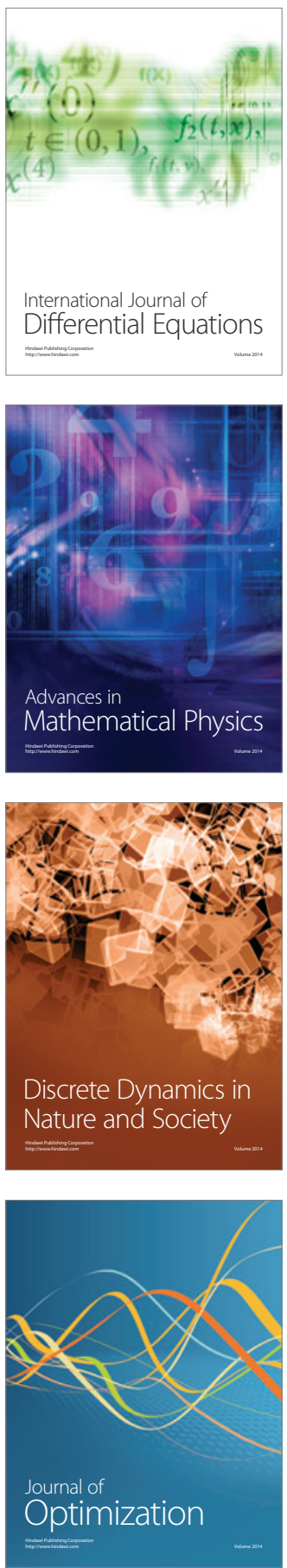